\title{
Mothers cafeteria diet induced sex-specific changes in fat content, metabolic profiles, and inflammation outcomes in rats offspring
}

Julia Matuszewska

Poznan University of Life Sciences

Tomasz Zalewski

Adam Mickiewicz University

Adam Klimaszyk

Adam Mickiewicz University

Kamil Ziarniak

Poznan University of Life Sciences

Stefan Jurga

Adam Mickiewicz University

Agata Chmurzynska

Poznan University of Life Sciences

Joanna H. Sliwowska ( $\nabla$ joanna.sliwowska@up.poznan.pl )

Poznan University of Life Sciences

\section{Research Article}

Keywords: obesity, cafeteria diet, prenatal programming, inflammation, sex-specific differences

Posted Date: August 20th, 2021

DOl: https://doi.org/10.21203/rs.3.rs-653347/v1

License: (c) (i) This work is licensed under a Creative Commons Attribution 4.0 International License. Read Full License 


\section{Abstract}

"Western diet" containing high concentrations of sugar and fat consumed during pregnancy contributing to development of obesity and diabetes type 2 in offspring. To mimic effects of this diet, in animals cafeteria (CAF) diet is used.

We hypothesis that CAF diet given to rats before, during pregnancy and lactation differently influence fat content, metabolic and inflammation profiles in offspring. Female were exposed to CAF or control diets before pregnancy, during pregnancy and lactation. At postnatal day 25 (PND 25) body composition, fat contents were measured, blood was collected for assessment of metabolic and inflammation profiles.

We have found that CAF diet leaded to sex-specific alterations in offspring. At PND25, CAF offspring had: 1) higher percentage of fat content and were lighter; 2$)$ sex-specific differences in levels of glucose; 3 ) higher levels of interleukins 6 (IL-6), interleukin-10 (IL-10) and tumor necrosis factor (TNF-a); 4) sexspecific differences in concentration of IL- 6 and TNF- $a$, with increase in CAF females; 5) higher level of IL10 in both sex, with bigger increase in females. We concluded that maternal CAF diet affects fat content, metabolic profiles, and inflammation parameters in offspring. Above effects are sex-specific, with female offspring being more susceptible to the diet.

\section{Introduction}

Obesity result from energy imbalance, which is often associated with of increased calorie intake and reduction in physical activity. Macronutrient composition of diets contribute to development of obesity ${ }^{1}$. So-called "Western diet" consumed mostly in highly developed countries is an example of unhealthy eating habits. It is characterized by high intakes of, butter, fried foods, high-fat dairy products, eggs, refined grains, potatoes and high-sugar drink ${ }^{2}$. To imitate effects of such diet in laboratory settings cafeteria (CAF) diet is used. It consists of variety of products, which are characterized by good taste with high energy density with about $45-55 \%$ of the energy comes from fats ${ }^{3-5}$. Commonly used ingredient is this diet are: biscuits, crisps, peanut butter, chocolate, chocolate bars, cheese, sausage, jam, muffins, cakes, dried fruit ${ }^{3,5,6}$. CAF diet very well mimic "fast food meals" popular among people ${ }^{7}$. It leads to development of obesity, characterized by increased body weight, changes in metabolic profiles and inflammation ${ }^{3-5}$.

Influence on "unhealthy diet" can start already in utero and according the prenatal programming theory environmental factor such as diet of mother influence fetus during its prenatal development and reprogramming neuroendocrine system. As results it may lead to the development of obesity, diabetes type 2 (DM2) and cardiovascular diseases. A strong correlation between low birth weight, high cortisol levels and later development of hypertension and DM2 8,9 . The offspring of mothers who suffer from diabetes during pregnancy are at higher risk of developing obesity and abnormal glucose metabolism not only in childhood but also during adolescence and adulthood ${ }^{10}$. Exposure to unhealthy diets can lead to 
remodeling and changes in offspring many organs. However, these effects are dependent on the composition of the obesogenic diet, its duration and timing of exposure and sex of offspring ${ }^{11,12}$.

In the light of above evidences, effective methods of assessment and control of body weight and composition are invaluable. Non-invasive techniques such as magnetic resonance image (MRI) ${ }^{13-18}$ and nuclear magnetic resonance (NMR) are used ${ }^{19-21}$ to study these changes. Above methods allow to examine abnormal fat contents, fat thickness as well as body composition, and help in prediction of development of metabolic disorders, which may appearances later in life ${ }^{22}$.

Similar to people consuming "Western type of diet", in adult animals CAF diet promotes compulsive food intake and rapid weight gain leading, increase fat content and induction of obesity ${ }^{5,23-25}$. Mice kept for 15 weeks on CAF diet had higher body weight and abdominal fat content when compared not only to control, but also to animals fed high-fat (HF) diet ${ }^{25}$. CAF diet also results in alterations of metabolic profile, induces lipogenesis and decreases very-low-density lipoprotein (VLDL) export leading to lipid accumulation in the liver and hepatic steatosis ${ }^{26-29}$. These changes are accompanied by increased concentrations of cholesterol, glucose, and triacylglycerol in blood 24,30-32. Finally, CAF diet leads to abdominal and visceral fat accumulation and insulin resistance $24,27,30$. In animal models, similar to situation in obese individuals, CAF diet-induced obesity results in inflammation $25,33,34$.

Exposure to CAF diet during pregnancy and/or lactation also influence body weight and metabolic profile of offspring. Administration of CAF diet to mother for 10 to 15 weeks altered body weight, elevated glucose, insulin, leptin and triglycerides levels ${ }^{35-37}$. Moreover, sex specific differences in levels of glucose and insulin in offspring after exposure of dams to CAF diet were found ${ }^{35-37}$ Yet, results of studies are not consistent and depends on many variables such as duration of the diet, its content, time of exposure, species and strain of animals.

Moreover, pups from mothers consuming CAF diet during lactation had lower body weight and lean mass, but greater fat accumulation, compared to controls at 3 months of age. Thus, it was proposed that feeding mothers CAF diet leads to a thin-outside-fat-inside phenotype in the offspring ${ }^{37}$. Similarly, at weaning (PND 21) rat offspring from mothers kept on CAF diet before pregnancy, during pregnancy and lactation had lower body weight but higher percentage of body fat content compared to control ${ }^{38}$. Additionally, both male and female CAF offspring being fed for 6 weeks after weaning control diet, remained lighter than control, however there was no longer difference in percentage of fat mass between groups ${ }^{38}$. However, in another experimental paradigm, when CAF diet was given for a longer time to rats before pregnancy, during pregnancy and lactation and post-weaning to offspring, there was no increase in body weight at puberty (PND 30), but animals had higher weigh at adulthood (PND 120) and no difference in visceral adipose tissue weight was reported in male rats ${ }^{6}$. Thus, differences in discussed parameters depends on duration of diet exposure as well as sex of offspring. 
Maternal obesity is also associated with a state of chronic, low-grade inflammation, characterized by elevated adipose tissue and systemic proinflammatory cytokine levels and adipose tissue macrophage accumulation ${ }^{39}$. These changes extend to the placenta, which suggest that maternal obesity exposes the fetus to an inflammatory environment during its development ${ }^{40-43}$. Indeed, numerous investigators reported changes in cytokines in the maternal and fetal/placental compartments (for review see ${ }^{39}$ ).

CAF diet also leads to inflammatory process in adult rodents. Fifteen weeks administration of CAF diet elevated serum level of interleukin 6 (IL-6) in male mice ${ }^{25}$, and administration of Western diet for 18 weeks to male rats increases level of tumor necrosis factor a (TNF-a) and decreased levels of IL-10 ${ }^{44}$. On the other hand, shorter exposure (for 6 weeks) to CAF diet to had no influence on inflammatory parameters (such as concentration of TNF-a and IL-6) in male rats ${ }^{34}$.

Effects of prenatal exposure to CAF diet on inflammatory processes were also studied ${ }^{45,46}$. CAF diet given to females before and during pregnancy and lactation increased IL- 6 cytokine, decrease expression of IL-1b and had no effects on TNF-a mRNA expression in the nucleus accumbens (NAc) shell of the brain in male offspring ${ }^{46}$. However, another study shown that feeding with CAF diet 8 weeks before prepregnancy and during pregnancy increased IL- 6 expression in placenta but did not alter inflammatory markers in the liver of fetuses on day 21 of pregnancy ${ }^{45}$.

Together, studies showed that female CAF diet consumed before and during pregnancy and/or lactation influence metabolic and inflammatory profiles and body composition of their offspring. However, there is only few reports on sex specific differences in effects of diet in offspring and data are contradictory ${ }^{35-37,}$ 47.

We hypothesis that rats fed CAF diet before and during pregnancy and during lactation differently influence metabolic profiles, fat content and inflammation parameters in males and females offspring.

\section{Results}

\section{Developmental data}

CAF diet in mothers increased food intake during pregnancy but did not altered body weight and fat content before, during and after pregnancy.

There were significant differences in food intake in mothers in second and third weeks of administration of CAF diet before pregnancy (p's $<0.05$; Fig. 2a). We have found that during these weeks mothers from CAF group ate more compared to $\mathrm{C}$ group. Moreover, there was difference in food intake in second week of pregnancy, when CAF mothers ate more compared to $C$ females $(p<0.05 ;$ Fig. $2 b$ ). We have also reported that after six weeks of CAF diet females were heavier compared to controls $(p<0.05)$ The same trend was seen in week seven $(p=0.06)$ (Fig. 2c). 
In terms of fat content, at the be beginning of experiment - first week $C$ females had higher fat content than CAF, but at the week 13th CAF females had higher fat contents compared to $C$ animals $(p<0.05$; Fig. 2d).

\section{Effect of CAF diet on fat volume in mothers before and after pregnancy and lactation}

CAF diet did not influence body weigt of pubs on PND 3 but leaded to sex-specific alterations in body weigh in offspring and fat content on PND 25

There were no differences in offspring weight on PND 3 between groups (CAF and $C$ ) in both sexes (females: for CAF $7.4 \mathrm{~g} \pm 0.3$ for C $7.8 \mathrm{~g} \pm 0.3$; males: for CAF $8.8 \mathrm{~g} \pm 0.8$, for C $7.5 \mathrm{~g} \pm 0.8$; $p$ 's $=0.11$; Figs. 4a and b, respectively). However, on PND 25 both male and female offspring from mothers kept on CAF diet had lower weight compared to control. Additionally, we found a marked sex differences in body weight of pups. Females offspring from CAF group weight about $20 \%$ less compared to controls (for CAF: $51.8 \mathrm{~g} \pm 2.9$; for C: $64.4 \mathrm{~g} \pm 2.9$; p's $<0.001 ; \mathrm{n}=9-13$; Fig. $4 \mathrm{c}$ ). Whereas, males offspring from CAF group weight about $16 \%$ less then these from C group (for CAF: $52.7 \mathrm{~g} \pm 4.0$; for C: $63.0 \mathrm{~g} \pm 4.0$; p's < 0.05; $n=7-10$; Fig. 4d).

There were also significant differences in percentage of fat in offspring on PND 25. Both female and male offspring from mothers kept on CAF diet had higher percentage fat compared with controls (females: for CAF $24.1 \mathrm{~g} \pm 1.3$, for C $12.8 \mathrm{~g} \pm 1.3, \mathrm{n}=9-13$; males: for CAF $22.5 \mathrm{~g} \pm 1.5$, for $\mathrm{C} 12.7 \mathrm{~g} \pm 1.5, \mathrm{n}$ = 7-10; p's $<0.001$; Figs. 5a, b, respectively).

\section{CAF diet altered metabolic parameters in offspring on PND 25 in sex-specific matter}

Females, but not in males offspring from mothers kept on CAF diet had a $14.5 \%$ higher blood glucose level when comparing to respective $\mathrm{C}$ groups (females: for CAF $141.8 \mathrm{mg} / \mathrm{dl} \pm 7.5$, for C $123.8 \mathrm{mg} / \mathrm{dl} \pm 7.5$; p's < 0.05; Fig. 6a; males: for CAF $143.8 \mathrm{mg} / \mathrm{dl} \pm 9.1$, for C $126.0 \mathrm{mg} / \mathrm{dl} \pm 9.1 ; \mathrm{p}=0.07$; Fig. $6 \mathrm{~b} ; \mathrm{n}=$ 5/sex/group).

In contrast, to glucose data, both females and males offspring from CAF mothers had a robust increase in level of insulin in blood serum compared to respective $\mathrm{C}$ groups. Additionally, we have reported that these differences were more pronounced in females. There was 8 times increase in this hormone levels in females CAF offspring, whereas in males there was 4,5 times rise, compared to relative controls offspring (females: for CAF $2.4 \mathrm{ng} / \mathrm{mL} \pm 0.3$, for C $0.3 \mathrm{ng} / \mathrm{mL} \pm 0.3 ; \mathrm{p}<0.005$; Fig. $6 \mathrm{c}$; males: for CAF $1.8 \mathrm{ng} / \mathrm{mL} \pm$ 0.3 , for $C 0.4 \mathrm{ng} / \mathrm{mL} \pm 0.3 ; \mathrm{p}<0.01$; Fig. $6 \mathrm{~d} ; \mathrm{n}=5$ / sex/group).

\section{CAF diet had a sex-specific effects on inflammatory parameters in offspring on PND 25}


We have also observed a sex-specific effects of mothers CAF diet on inflammatory parameters in offspring. There were differences in IL-6 level in blood serum in offspring from mothers kept on CAF diet, when compared to $\mathrm{C}$ group. However, these differences reach statistically significant values in females (14\% increase), but not in males offspring (females: for CAF $65.0 \mathrm{pg} / \mathrm{ml} \pm 8.3$, for C $41.8 \mathrm{pg} / \mathrm{ml} \pm 8.3 ; \mathrm{p}<$ 0.05; Fig. 7a; males: for CAF $60.7 \mathrm{pg} / \mathrm{ml} \pm 9.8$, for C $43.9 \mathrm{pg} / \mathrm{ml} \pm 9.8 ; \mathrm{p}=0.12$; Figs. $7 \mathrm{~b} ; \mathrm{n}=5 /$ sex/group).

In contrast to IL-6, both female and male offspring from mothers kept on CAF diet had significantly higher concentration of IL-10 in blood serum compared to respective $\mathrm{C}$ groups. However, again we found that this increase was bigger in females, compared to males (females: 3,5 times increase; for CAF 376.8 $\mathrm{pg} / \mathrm{mL} \pm 40.4$, for C $107.8 \mathrm{pg} / \mathrm{mL} \pm 40.4$; $\mathrm{p}<0.005$; Fig. 7c; males: 3,2 times rise; for CAF $265.6 \mathrm{pg} / \mathrm{mL} \pm$ 28.3, C $82.5 \mathrm{pg} / \mathrm{mL} \pm 28.3 ; \mathrm{p}<0.005 ;$ Fig. $7 \mathrm{~d} ; \mathrm{n}=5$ /sex/group).

Finally, similar to IL- 6 data, level of TNF-a in blood serum were significantly higher (2,7 times) at in females but not males offspring from mothers kept on CAF diet when compared to $\mathrm{C}$ groups. In females, there was $62 \%$ increase compared to respective controls (females: for CAF $218.5 \mathrm{pg} / \mathrm{ml} \pm 23.9$, for C 82.3 $\mathrm{pg} / \mathrm{ml} \pm 23.9 ; \mathrm{p}<0.005$; Fig. 7e; males: for CAF $127.8 \mathrm{pg} / \mathrm{ml} \pm 26.1$, for C $91.5 \mathrm{pg} / \mathrm{ml} \pm 26.1 ; \mathrm{p}=0.20$; Fig. $7 f ; n=5 /$ sex/group).

\section{Discussion}

We confirmed the hypothesis that mothers cafeteria diet differently influence metabolic profiles, fat content and inflammation parameters in males and females rat offspring. Besides study of pups, we also examined changes in females before during pregnancy and during lactation. We found that dams on CAF diet had increased food intake in second and third week before pregnancy and in second week of pregnancy when compared to $\mathrm{C}$ group. These findings are in agreement with data obtained by Sanchez-Blanco et al., which shown that female rats kept on CAF diet 22 days before pregnancy and during pregnancy had higher food intake then control ${ }^{48}$.

In our study, in spite of reported here increase in food intake in dams, no statistically significant differences in mothers' weight and fat results were noticed. But, inspection of figures (Figs. 3a and b) indicate a non-significant increase in body weight in dams. These, non-significant effects maybe a result of small study groups ( $n=3$ mothers/ group). Indeed, studies on bigger number of dams ( $n=16 /$ group) shown that 6-13 weeks of exposure to CAF diet rats induced increase in weight in dams ${ }^{36}$. Additionally, it was found that CAF rats gained an extra $22 \%$ in weight during the pre-pregnancy period compared to controls, and were heavier during pregnancy. Moreover, CAF diet fed dams still remained heavier than animals fed a chow diet at mid-lactation ${ }^{36}$.

Our studies also confirmed that both MRI and NMR are useful, non-invasive techniques in assessment of fat contents and body composition, which could be successfully employed in studies of effects of CAF diet on development of obesity. However, one limitation of MRI study in anesthetized animal is heart and breathing movements, which unable to examine abdominal fat. Thus, in this study we could only 
examined volume of fat tissue on the neck of animals. Here due to logistic considerations, we were not able to perform MRI studies in pups. But, in the future we plan experiments using MRI on pups to examine changes in fat content at different time points of postnatal development.

The main aim of this study was however to examine influence of the CAF diet on pups and study possible sex differences in response to this metabolic insult.

We have found that while on PND 3 there was no difference in body weight between pups on CAF and C diets. Such differences were revealed by PND 25 when both males and females offspring from mothers kept on CAF diet had lower weight compared to control. Additionally, we have found that at PND 25 both pups of both sex from mother kept on CAF diet had higher fat contents. Thus, in our study we have confirmed reported by Pomar et al. ${ }^{37}$ thin outside, fat inside phonotype in pups. Moreover, we have revealed that these differences were more pronounced in females offspring.

Cardenas-Perez et al. shown that offspring from mothers kept on CAF diet for 9 weeks (including premating, pregnancy and lactation) had slightly lower body weight at birth comparing to $\mathrm{C}$ group ${ }^{49}$. Moreover, when offspring were fed CAF diet after weaning, this decrease in body weight persist until week 7 of age. Study performed by Sanchez-Blanco ${ }^{48}$ body weight at birth in pups from mothers kept of CAF diet 22 days before pregnancy and during pregnancy was also lower, compared to control. Additionally, when pups remained on CAF diet continue to be lighter until PND $21^{48}$. Similarly, in experiments conducted by Bayol ${ }^{35}$ revealed that when CAF diet was given during gestation and lactation to mothers it caused a decrease in body weight of offspring both at birth and at weaning (PND 21). However, when such diet was continuing up to week 10 postnatally an increase in body weigh both in males and females was reported ${ }^{35}$. Thus, our data and discussed above studies indicate that CAF diet given to mothers before pregnancy, during pregnancy and lactation leads to decrease in body weight in offspring. Lower body weight of offspring exposed prenatally to CAF diet may be related to decrease protein intake by approximately $37 \%$ and $34 \%$ during gestation and lactation, respectively ${ }^{50}$.

Our study also shown that in CAF females' offspring at PND 25 decrease in body weight is more pronounced compared to CAF males' offspring. Sex differences in body weight of offspring was also reported in experiment when mixture of high fat (HFD) and CAF diets (HFD + CAF) were given before mating, and during pregnancy to rats ${ }^{51}$. Males but not females' offspring from HFD + CAF groups had lower weight on PND 1. However, on PND 19 both male and female offspring form mothers on HFD + CAF diet, which remained on such diet after delivery, had higher body weight compared to $\mathrm{C}$ group ${ }^{51}$.

Numerous experiments including our current study also confirm that exposure to CAF diet: 1) before pregnancy and during pregnancy and lactation, 2) during pregnancy and lactation, 3) only during lactation leads to accumulation of fat tissue in offspring $37,38,52,53$.

Sex-specific differences were also found in blood glucose and insulin levels. On PND 25 only in females from CAF mothers was significant increase in blood glucose level. However, in case of insulin, 
concentration of this hormone was statistically significant higher both in males and females offspring form mother kept on CAF diet. But, again this rise in insulin level was more pronounced in females offspring.

George et al. shown, that both sexes of Wistar rats offspring at age of 12 weeks from mothers kept on CAF diet during pregnancy had not changes in fasting serum glucose levels. However, when dam was exposed to CAF during lactation offspring had significant higher level of fasting serum glucose comparing to control ${ }^{36}$. Moreover, only male offspring from mothers kept on CAF diet during lactation had higher pick of glucose in glucose tolerance test at 12 weeks ${ }^{36}$. Pomar et al., also shown that offspring at 3 and 6 weeks of age from mothers kept on CAF diet during lactation had impaired response to glucose tolerance test ${ }^{37}$. Furthermore, study of George ${ }^{36}$ and Pomar ${ }^{37}$ found no difference in plasma insulin levels between CAF and C offspring. However, George et al. ${ }^{36}$ shown that overall male offspring had significant higher level of plasma insulin compared with female, particularly at baseline and two hours. Thus, the Authors confirm previous finding that exposure during lactation to CAF diet causing male offspring to be more susceptible to insulin resistance ${ }^{54,55}$. It was also concluded that only postnatal exposure to maternal obesity lead to adiposity and insulin resistance. It was proposed that the milk composition of the dams is key factor for programming effects. In our study offspring remains on CAF diet while in utero as well as during lactation, which prevent us to dissociate between prenatal and postnatal effects of the diet. Additionally, we have studied offspring in much younger age (PND 25). However, similar to above mentioned studies we also revealed sex-specific effects of CAF diet on metabolic outcomes.

Effects of CAF diet on glucose levels were also studied in offspring. Bayol et al. revealed that exposure of Wistar rats to CAF diet during pregnancy, lactation and post-weaning period leads to sex differences in both glucose and insulin levels in offspring ${ }^{35}$. Similar to our results at PND 25, females at age 10 weeks from mother on CAF diet had higher concentration of glucose level when compared to control ${ }^{35}$. However, both in Bayol ${ }^{35}$ and our studies there was no difference in glucose levels between males offspring form CAF and $\mathrm{C}$ mothers. On the other hand, concentration of insulin was significant higher in male offspring in comparison to control, but with no difference in female offspring at age of 10 weeks ${ }^{35}$. In our study, with shorter exposure to CAF diet, raised level of this hormone was seen both in male and female offspring. Thus, additional feeding post weaning with CAF diet may contribute to increased levels of insulin observed in males in age of 10 weeks. However, study performed by Bayol ${ }^{35}$ also indicate that CAF -induced adiposity and metabolic disruptions were increased in adult offspring from mothers exposed to such diet in pregnancy and lactation when compared with offspring only fed the diet after weaning. Indeed, it was shown that adipocyte hypertrophy and increase in perirenal fat pad mass relative to body weight persist, even when the offspring were fed a chow diet after weaning.

Here we have provided novel finding on sex-specific differences in inflammatory parameters in offspring in response to maternal CAF diet. Significant increase of interleukin 6 (IL-6) and tumor necrosis factor (TNF-a) was found in females from CAF mothers, but not in males offspring. In contrast, significant 
elevation of interleukin 10 (IL-10) was found in both sexes of offspring. However, concentration of IL-10 in females from CAF mothers was higher than in male offspring. Our results are in agreement with studies performed on adult rats exposed to CAF diet. CAF diet leads to TNF-a overexpression in the intestine ${ }^{56}$ and in plasma ${ }^{33}$ in adult male rats ${ }^{5}$. CAF-fed adult rats also displayed remarkable inflammation in white fat, brown fat and liver ${ }^{33}$. Both in animal and human obesity low-level chronic inflammation and macrophage infiltration into adipose tissue is a well-documented phenomenon ${ }^{57-59}$. However, signs of inflammation were found not only at the periphery but also centrally, as consumption of CAF diet increases both peripheral and central levels of interleukin-1 $\beta$ (IL-1 $\beta)$, a pro-inflammatory cytokine. Eighteen weeks of administration of Western diet elevated blood serum level of TNF- $\alpha$ and decreased levels of IL-10 and IL-6 in in male Wistar rats, when compared to control ${ }^{44}$. However, shorter 6 weeks of administration of CAF diet to male adult rats had no significant influence of diet on inflammatory parameters (TNF-a and IL-6) ${ }^{34}$. But, longer administration of CAF diet (15 weeks) to adult mice leaded to inflammatory processes, characterized by significant higher concentration of IL- $6^{25}$. Thus, duration of administration of CAF diet maybe a key parameters when discussing inflammatory responses. In contrast to well documented inflammation caused by CAF diet in adult animals, literature on this subject in prenatal models is spare. When CAF diet was given to rat mothers 8 weeks before pregnancy and during pregnancy it did not increase the inflammatory status of the mother, placenta or fetus in late gestation (PND 21) ${ }^{45}$. Actually, levels of inflammatory markers such as IL-6, IL-12p40 and MIP2 were reduced slightly. Carillon et al. shown that Wistar rats offspring from mothers kept on CAF diet for 9 weeks, including pregnancy and lactation had higher mRNA expression of IL- 6 in brain than C. However, differences in TNF-a mRNA expression was not observed ${ }^{23}$.

In spite of that CAF diet is commonly used in many laboratories, the main caveat refers to its nutritional composition, which is uncontrolled, given that the animals can choose among a variety of different foods 60 . There is no standard CAF diet protocol, its caloric content and selection of products varies largely between studies ${ }^{61}$. Additionally, percentage of energy derived from fat and carbohydrates differs and ranges between 17-60 and 37 and 73, respectively. Moreover, while in some studies CAF was used alone, in other it has been combined with standard chow diet. However, one way to overcome these difficulties is to simplified the diet, and employed with only 3 or 4 food choices ${ }^{62,63}$, which were used in the current study. Finally, protocols used by different researches varied in time of exposure to the diet.

In summary, we have shown that mothers exposure to CAF diet, which mimic "Western-style diet" before and during pregnancy and lactation increased body weight, fat contents, changed metabolic profiles (levels of glucose and insulin), and led to inflammation in offspring. Moreover, we have revealed that above alterations are sex-specific and more pronounced in female offspring. We have also shown that non-invasive imaging techniques such as NMR and MRI could be successfully employed in CAF-induced obesity model. Our study indicates a crucial need to monitor early sign of development of metabolic problem in offspring form mothers kept on CAF diet with respect to sex differences.

\section{Materials And Methods}


Six 2-months-old female Wistar rats ( $300 \mathrm{~g} \pm 13 \mathrm{~g}$ ) and three 2 -months-old male Wistar rats were obtained from licensed Animal Breeding Company in Poznan. Rats were housed in cages under constant conditions of light/dark cycle $12 / 12$ hours and temperature $\left(21^{\circ} \mathrm{C}\right)$. Animals had ad libitum access to water and standard laboratory chow diet (AIN93G, Zoolab, Poland). After one week of acclimatization rats were divided into two groups: 1 ) cafeteria diet (CAF; $n=3$; animals fed with composed diet; see Table 1 for used products and their nutritional values) and control $(C ; n=3$; animals fed standard chow diet AIN93G, Zoolab, Polska). Females from CAF group were fed CAF diet for four weeks before pregnancy, as well as during pregnancy and lactation. Similarly, standard chow diet were administrated to $\mathrm{C}$ group for the same amount of time. Both groups had ad libitum access to water and food during experiment (Fig.1). Experiment was approved by the Local Ethics Committee for Experiments on Animals, Poznan University of Life Sciences, Poland (license no. 55/2018; 38/2020). All experiments were performed in accordance with relevant guidelines and regulations. Reporting in the manuscript follows the recommendations in the ARRIVE guidelines. After 4 weeks of administration of CAF or C diet, six adult females were paired with three adult males for fertilization. Male and female were kept in wire-bottom cage for two days. Pregnancies were confirmed by presence of vaginal plugs. After fertilization females were kept in separate cages during whole pregnancy and lactation with ad libitum access to food, water and nest material (Fig. 1).

Composition of cafeteria ( $\underline{\mathrm{CAF}})$ diet

CAF diet were composed by experimenter based on earlier published studies using that kind of diet ${ }^{3,24}$. CAF diet consists of 12 different products: cheese, dried sausage, biscuits, chocolate, peanut butter, chips, cookies, pate, jam, chocolate bar, dried bananas and muffin (Table 1). Products and their nutritional values in CAF diet are presented in Table 1. Every day each female from CAF diet group received three products (two sweet and one savoury). Food portions were weight on laboratory scale and products were changed daily and in threes, so that these three do not repeat. Rats had ad libitum access to CAF diet and tab water during the whole experiment. Remaining food was weight daily and food intake was calculated.

\section{Body composition test}

Females from both groups (CAF and $\mathrm{C}$ ) were analyzed weekly (except pregnancy and lactation period) by nuclear magnetic resonance (NMR) using Minispec LF90 (Billerica, Massachusetts, USA). It is noninvasive and short time analyses, in which weight of animal as well as a whole animal body composition (absolute weight of fat and free body liquid) are measured. NMR technique does not require administration of anesthetics, which makes it safety and not stressful for animals. Obtained from NMR data were analyzed and percentage of fat content in each time point were calculated.

\section{MRI scanning}


After four weeks of administration of diets dams from CAF and C groups were subjected to MRI scanning. MRI experiment were carried out using a preclinical horizontal scanner operating at 9,4 T (400 $\mathrm{MHz}$ - Agilent) equipped with a $600 \mathrm{mT} / \mathrm{m}$ gradient system. For MRI imaging a 72-mm i.d. quadrature birdcage type coil was used.

During the MRI experiment animals were put at special designed holder and anesthetized with $1.5-2 \%$ isoflurane in a 50/50 air-oxygen mixture. The temperature of the animal was kept at $37^{\circ} \mathrm{C}$. Respiration of the animal were monitoring and used to synchronize MRI experiments.

MRI images of spin density were collected at the location of fat tissue using fast spin echo technique (FSEMS) with parameters: TR=5s, effective TE=10 ms, ETL = 8, FOV $62 \times 62 \mathrm{~mm}$, matrix size 256x256, 18 slices. Next at the same location FSEMs sequence with additional fat saturation was used.

Collected data with and without fat signal were transformed to DICOM format and obtained images from second experiment were subtracted from collected images using ImageJ software. Then the Volumest (ImageJ plugin) where employed to count volumes of fat for every animal in three independent trials.

\section{Offspring}

Pups were born at 22-23 day of pregnancy. On postnatal day 3 (PND 3) pups were weight on laboratory scales and sex was identified. Sex checked was guided by distance between anus and urethra. On PND 3 litters were unification to 8 pups per litter.

\section{Sample collection}

On PND 25 animals were analyzed by NMR to measured body composition (weight, absolute fat and free body liquid content). Then animals were sacrificed by using $\mathrm{CO}_{2}$ chamber (Equipement Veterinaire Minerve, Esternay, France), decapitated, trunk blood was collected and blood serum was frozen in liquid nitrogen, and stored at $-80^{\circ} \mathrm{C}$ for further testing. Because of the small amount, blood serum of offspring was pooled to $n=5 /$ sex $/$ group.

\section{Metabolic profiles of offspring}

Levels of glucose in pups were measured by AccuCheck Active device (Roche Diabetes Care, Warsaw, Poland). Offspring insulin level in blood serum was measured by radioimmunoassay rat RIA kit (Rat insulin RIA test RI-13K, Merck).

\section{Inflammatory_profiles of offspring}

Levels of interleukins: IL- 6 and IL-10 as well as tumor necrosis factor alfa -TNF-a in blood serum were measured using immune-enzymatic ELISA kits (Elabscience, Wuhan, Hubei, China).

Statistical analysis and data presentation 
Mothers body weight fat content, food intake were measures using analysis of variance (ANOVA) for the factors of group (CAF and C), with day as a repeated measure. Volume mother of fat tissue, offspring weight, fat contents, metabolic and inflammatory parameters were analyzed using unpaired t-test, compering CAF and $\mathrm{C}$ groups. For measurement of fat volume from MRI scans Image J software (https://imagej.nih.gov/ij/) and Volumest (ImageJ plugin) were used. Graphs were prepared using Graph Pad Prism 8 software (GraphPad Software, San Diego, USA). Data are presented as means \pm SEM. Pvalue of less than 0.05 was considered statistically significant. All statistical analyses were performed using Graph Pad Prism 8 software (GraphPad Software, San Diego, USA).

\section{Bibliography}

1. Abbasi, J. Study Puts Eggs and Dietary Cholesterol Back on the Radar. JAMA, 321, 1959-1961 https://doi.org/10.1001/jama.2019.4165 (2019).

2. Halton, T. L. et al. Potato and french fry consumption and risk of type 2 diabetes in women. $A m \mathrm{~J}$ Clin Nutr, 83, 284-290 https://doi.org/10.1093/ajcn/83.2.284 (2006).

3. Jacobs, S. et al. The impact of maternal consumption of cafeteria diet on reproductive function in the offspring. Physiol Behav, 129, 280-286 https://doi.org/10.1016/j.physbeh.2014.03.003 (2014).

4. Rothwell, N. J., Saville, M. E. \& Stock, M. J. Effects of feeding a "cafeteria" diet on energy balance and diet-induced thermogenesis in four strains of rat. J Nutr, 112, 1515-1524 https://doi.org/10.1093/jn/112.8.1515 (1982).

5. Sampey, B. P. et al. Cafeteria diet is a robust model of human metabolic syndrome with liver and adipose inflammation: comparison to high-fat diet. Obesity (Silver Spring), 19, 1109-1117 https://doi.org/10.1038/oby.2011.18 (2011).

6. Mucellini, A. B. et al. Effects of exposure to a cafeteria diet during gestation and after weaning on the metabolism and body weight of adult male offspring in rats. Br J Nutr, 111, 1499-1506 https://doi.org/10.1017/S0007114513003838 (2014).

7. Bridge-Comer, P. E., Vickers, M. H. \& Reynolds, C. M. Preclinical Models of Altered Early Life Nutrition and Development of Reproductive Disorders in Female Offspring. Adv Exp Med Biol, 1134, 59-87 https://doi.org/10.1007/978-3-030-12668-1_4 (2019).

8. Phillips, D. I. et al. Elevated plasma cortisol concentrations: a link between low birth weight and the insulin resistance syndrome? J Clin Endocrinol Metab, 83, 757-760 https://doi.org/10.1210/jcem.83.3.4634 (1998).

9. Phillips, D. I. et al. Low birth weight predicts elevated plasma cortisol concentrations in adults from 3 populations., 35, 1301-1306 https://doi.org/10.1161/01.hyp.35.6.1301 (2000).

10. Chavatte-Palmer, P., Tarrade, A. \& Rousseau-Ralliard, D. Diet before and during Pregnancy and Offspring Health: The Importance of Animal Models and What Can Be Learned from Them. Int J Environ Res Public Health, 13, https://doi.org/10.3390/ijerph13060586 (2016). 
11. Langley-Evans, S. C. Nutrition in early life and the programming of adult disease: a review. $J$ Hum Nutr Diet, 28 (Suppl 1), 1-14 https://doi.org/10.1111/jhn.12212 (2015).

12. Li, M., Sloboda, D. M. \& Vickers, M. H. Maternal obesity and developmental programming of metabolic disorders in offspring: evidence from animal models. Exp Diabetes Res 2011, 592408, doi:10.1155/2011/592408 (2011).

13. Cherbuin, N., Sargent-Cox, K., Fraser, M., Sachdev, P. \& Anstey, K. J. Being overweight is associated with hippocampal atrophy: the PATH Through Life Study. Int J Obes (Lond), 39, 1509-1514 https://doi.org/10.1038/ijo.2015.106 (2015).

14. Kn, B. P., Gopalan, V., Lee, S. S. \& Velan, S. S. Quantification of abdominal fat depots in rats and mice during obesity and weight loss interventions. PLoS One, 9, e108979 https://doi.org/10.1371/journal.pone.0108979 (2014).

15. Mitra, S., Fernandez-Del-Valle, M. \& Hill, J. E. The role of MRI in understanding the underlying mechanisms in obesity associated diseases. Biochim Biophys Acta Mol Basis Dis, 1863, 1115-1131 https://doi.org/10.1016/j.bbadis.2016.09.008 (2017).

16. Mole, T. B., Mak, E., Chien, Y. \& Voon, V. Dissociated Accumbens and Hippocampal Structural Abnormalities across Obesity and Alcohol Dependence. Int J Neuropsychopharmacol, 19, https://doi.org/10.1093/ijnp/pyw039 (2016).

17. Ronn, M. et al. Quantification of total and visceral adipose tissue in fructose-fed rats using water-fat separated single echo MRI. Obesity (Silver Spring), 21, E388-395 https://doi.org/10.1002/oby.20229 (2013).

18. Schoettl, T., Fischer, I. P. \& Ussar, S. Heterogeneity of adipose tissue in development and metabolic function. J Exp Biol, 221, https://doi.org/10.1242/jeb.162958 (2018).

19. Reynolds, T. H. et al. The impact of age and sex on body composition and glucose sensitivity in C57BL/6J mice. Physiol Rep, 7, e13995 https://doi.org/10.14814/phy2.13995 (2019).

20. Wishart, D. S. Quantitative metabolomics using NMR. Trends in Analytical Chemistry27 (2008).

21. Zhang, S. et al. Correlative and quantitative $1 \mathrm{H}$ NMR-based metabolomics reveals specific metabolic pathway disturbances in diabetic rats. Anal Biochem, 383, 76-84 https://doi.org/10.1016/j.ab.2008.07.041 (2008).

22. Lemos, T. \& Gallagher, D. Current body composition measurement techniques. Curr Opin Endocrinol Diabetes Obes, 24, 310-314 https://doi.org/10.1097/MED.0000000000000360 (2017).

23. Carillon, J. et al. Cafeteria diet induces obesity and insulin resistance associated with oxidative stress but not with inflammation: improvement by dietary supplementation with a melon superoxide dismutase. Free Radic Biol Med, 65, 254-261 https://doi.org/10.1016/j.freeradbiomed.2013.06.022 (2013).

24. Gomez-Smith, M. et al. A physiological characterization of the Cafeteria diet model of metabolic syndrome in the rat. Physiol Behav, 167, 382-391 https://doi.org/10.1016/j.physbeh.2016.09.029 (2016). 
25. Zeeni, N., Dagher-Hamalian, C., Dimassi, H. \& Faour, W. H. Cafeteria diet-fed mice is a pertinent model of obesity-induced organ damage: a potential role of inflammation. Inflamm Res, 64, 501-512 https://doi.org/10.1007/s00011-015-0831-z (2015).

26. de Melo, A. F. et al. Increase in liver cytosolic lipases activities and VLDL-TAG secretion rate do not prevent the non-alcoholic fatty liver disease in cafeteria diet-fed rats., 150, 16-22 https://doi.org/10.1016/j.biochi.2018.04.021 (2018).

27. Feijo, G. D. S. et al. Food Selection of Cafeteria Diet Affects Memory Dysfunction Related to Obesity. Neurochem Res, 44, 1869-1877 https://doi.org/10.1007/s11064-019-02821-5 (2019).

28. Gasparin, F. R. S. et al. Sex differences in the development of hepatic steatosis in cafeteria dietinduced obesity in young mice. Biochim Biophys Acta Mol Basis Dis, 1864, 2495-2509 https://doi.org/10.1016/j.bbadis.2018.04.004 (2018).

29. Maeda Junior, A. S. et al. Cafeteria Diet Feeding in Young Rats Leads to Hepatic Steatosis and Increased Gluconeogenesis under Fatty Acids and Glucagon Influence. Nutrients, 10, https://doi.org/10.3390/nu10111571 (2018).

30. Gastiazoro, M. P. et al. Induction of uterine hyperplasia after cafeteria diet exposure. Mol Cell Endocrinol, 477, 112-120 https://doi.org/10.1016/j.mce.2018.06.007 (2018).

31. Lalanza, J. F. et al. Effects of a post-weaning cafeteria diet in young rats: metabolic syndrome, reduced activity and low anxiety-like behaviour. PLoS One, 9, e85049 https://doi.org/10.1371/journal.pone.0085049 (2014).

32. Warneke, W., Klaus, S., Fink, H., Langley-Evans, S. C. \& Voigt, J. P. The impact of cafeteria diet feeding on physiology and anxiety-related behaviour in male and female Sprague-Dawley rats of different ages. Pharmacol Biochem Behav, 116, 45-54 https://doi.org/10.1016/j.pbb.2013.11.016 (2014).

33. Gonzalez-Quilen, C. et al. Grape-Seed Proanthocyanidins are Able to Reverse Intestinal Dysfunction and Metabolic Endotoxemia Induced by a Cafeteria Diet in Wistar Rats. Nutrients, 11, https://doi.org/10.3390/nu11050979 (2019).

34. Navarro, M. E. et al. Renal inflammatory and oxidative and metabolic changes after 6 weeks of cafeteria diet in rats. J Bras Nefrol, 38, 9-14 https://doi.org/10.5935/0101-2800.20160003 (2016).

35. Bayol, S. A., Simbi, B. H., Bertrand, J. A. \& Stickland, N. C. Offspring from mothers fed a 'junk food' diet in pregnancy and lactation exhibit exacerbated adiposity that is more pronounced in females. $J$ Physiol, 586, 3219-3230 https://doi.org/10.1113/jphysiol.2008.153817 (2008).

36. George, G. et al. Exposure to maternal obesity during suckling outweighs in utero exposure in programming for post-weaning adiposity and insulin resistance in rats. Sci Rep, 9, 10134 https://doi.org/10.1038/s41598-019-46518-9 (2019).

37. Pomar, C. A. et al. Maternal consumption of a cafeteria diet during lactation in rats leads the offspring to a thin-outside-fat-inside phenotype. Int J Obes (Lond), 41, 1279-1287 https://doi.org/10.1038/ijo.2017.42 (2017).

38. Vithayathil, M. A. et al. Exposure to maternal cafeteria diets during the suckling period has greater effects on fat deposition and Sterol Regulatory Element Binding Protein-1c (SREBP-1c) gene 
expression in rodent offspring compared to exposure before birth. Nutr Metab (Lond), 15, 17 https://doi.org/10.1186/s12986-018-0253-3 (2018).

39. Pantham, P., Aye, I. L. \& Powell, T. L. Inflammation in maternal obesity and gestational diabetes mellitus., 36, 709-715 https://doi.org/10.1016/j.placenta.2015.04.006 (2015).

40. Basu, S. et al. Pregravid obesity associates with increased maternal endotoxemia and metabolic inflammation. Obesity (Silver Spring), 19, 476-482 https://doi.org/10.1038/oby.2010.215 (2011).

41. Challier, J. C. et al. Obesity in pregnancy stimulates macrophage accumulation and inflammation in the placenta., 29, 274-281 https://doi.org/10.1016/j.placenta.2007.12.010 (2008).

42. Madan, J. C. et al. Maternal obesity and markers of inflammation in pregnancy., 47, 61-64 https://doi.org/10.1016/j.cyto.2009.05.004 (2009).

43. Segovia, S. A., Vickers, M. H., Gray, C. \& Reynolds, C. M. Maternal obesity, inflammation, and developmental programming. Biomed Res Int 2014, 418975, doi:10.1155/2014/418975 (2014).

44. Bortolin, R. C. et al. A new animal diet based on human Western diet is a robust diet-induced obesity model: comparison to high-fat and cafeteria diets in term of metabolic and gut microbiota disruption. Int J Obes (Lond), 42, 525-534 https://doi.org/10.1038/ijo.2017.225 (2018).

45. Crew, R. C., Waddell, B. J. \& Mark, P. J. Maternal obesity induced by a 'cafeteria' diet in the rat does not increase inflammation in maternal, placental or fetal tissues in late gestation., 39, 33-40 https://doi.org/10.1016/j.placenta.2016.01.002 (2016).

46. Cruz-Carrillo, G. et al. Fetal Programming by Methyl Donors Modulates Central Inflammation and Prevents Food Addiction-Like Behavior in Rats. Front Neurosci, 14, 452 https://doi.org/10.3389/fnins.2020.00452 (2020).

47. Ingvorsen, C., Brix, S., Ozanne, S. E. \& Hellgren, L. I. The effect of maternal Inflammation on foetal programming of metabolic disease. Acta Physiol (Oxf), 214, 440-449 https://doi.org/10.1111/apha.12533 (2015).

48. Sanchez-Blanco, C., Amusquivar, E., Bispo, K. \& Herrera, E. Influence of cafeteria diet and fish oil in pregnancy and lactation on pups' body weight and fatty acid profiles in rats. Eur $J$ Nutr, 55, 17411753 https://doi.org/10.1007/s00394-015-0992-0 (2016).

49. Cardenas-Perez, R. E. et al. Maternal overnutrition by hypercaloric diets programs hypothalamic mitochondrial fusion and metabolic dysfunction in rat male offspring. Nutr Metab (Lond), 15, 38 https://doi.org/10.1186/s12986-018-0279-6 (2018).

50. Bayol, S. A., Farrington, S. J. \& Stickland, N. C. A maternal 'junk food' diet in pregnancy and lactation promotes an exacerbated taste for 'junk food' and a greater propensity for obesity in rat offspring. $\mathrm{Br}$ J Nutr, 98, 843-851 https://doi.org/10.1017/S0007114507812037 (2007).

51. Raipuria, M., Bahari, H. \& Morris, M. J. Effects of maternal diet and exercise during pregnancy on glucose metabolism in skeletal muscle and fat of weanling rats. PLoS One, 10, e0120980 https://doi.org/10.1371/journal.pone.0120980 (2015).

52. Bayol, S. A., Simbi, B. H. \& Stickland, N. C. A maternal cafeteria diet during gestation and lactation promotes adiposity and impairs skeletal muscle development and metabolism in rat offspring at 
weaning. J Physiol, 567, 951-961 https://doi.org/10.1113/jphysiol.2005.088989 (2005).

53. Ong, Z. Y. \& Muhlhausler, B. S. Maternal "junk-food" feeding of rat dams alters food choices and development of the mesolimbic reward pathway in the offspring. FASEB J, 25, 2167-2179 https://doi.org/10.1096/fj.10-178392 (2011).

54. Akyol, A., McMullen, S. \& Langley-Evans, S. C. Glucose intolerance associated with early-life exposure to maternal cafeteria feeding is dependent upon post-weaning diet. $\mathrm{Br} J \mathrm{Nutr}, 107,964-978$ https://doi.org/10.1017/S0007114511003916 (2012).

55. Gorski, J. N., Dunn-Meynell, A. A., Hartman, T. G. \& Levin, B. E. Postnatal environment overrides genetic and prenatal factors influencing offspring obesity and insulin resistance. Am J Physiol Regul Integr Comp Physiol, 291, R768-778 https://doi.org/10.1152/ajpregu.00138.2006 (2006).

56. Gil-Cardoso, K. et al. A cafeteria diet triggers intestinal inflammation and oxidative stress in obese rats. Br J Nutr, 117, 218-229 https://doi.org/10.1017/S0007114516004608 (2017).

57. Hotamisligil, G. S. Inflammation and endoplasmic reticulum stress in obesity and diabetes. Int J Obes (Lond) 32 Suppl 7, S52-54, doi:10.1038/ijo.2008.238 (2008).

58. Makowski, L. \& Hotamisligil, G. S. Fatty acid binding proteins-the evolutionary crossroads of inflammatory and metabolic responses. $J$ Nutr, 134, 2464-2468 https://doi.org/10.1093/jn/134.9.2464S (2004).

59. Rasouli, N. \& Kern, P. A. Adipocytokines and the metabolic complications of obesity. J Clin Endocrinol Metab, 93, S64-73 https://doi.org/10.1210/jc.2008-1613 (2008).

60. Moore, J. M. The cafeteria diet-An inappropriate tool for studies of thermogenesis. Journal of Nutrition, 117, 227-231 (1987).

61. Lai, M., Chandrasekera, P. C. \& Barnard, N. D. You are what you eat, or are you? The challenges of translating high-fat-fed rodents to human obesity and diabetes. Nutr Diabetes, 4, e135 https://doi.org/10.1038/nutd.2014.30 (2014).

62. Rogers, P. J. Returning 'cafeteria-fed' rats to a chow diet: negative contrast and effects of obesity on feeding behaviour. Physiol Behav, 35, 493-499 https://doi.org/10.1016/0031-9384(85)90129-5 (1985).

63. Sclafani, A., Aravich, P. F. \& Landman, M. Vagotomy blocks hypothalamic hyperphagia in rats on a chow diet and sucrose solution, but not on a palatable mixed diet. J Comp Physiol Psychol, 95, 720734 https://doi.org/10.1037/h0077830 (1981).

\section{Declarations}

\section{Acknowledgements}

We would like to thank Małgorzata Tubacka, Ewelina Żuk, Anna Radziejewska and Anna Makuch for technical assistance with animal experiment. Adam Klimaszyk would like to acknowledge the project No. POWR.03.02.00-00-1032/16. 


\section{Funding}

The study was support by research task as part of a specific subsidy for tasks supporting the development of young scientists and participants of doctoral studies 506-511-09-00 and by the National Science Center in Krakow, Poland (grant PRELUDIUM 2019/35/N/NZ9/00663 to JM) and by SJ\&TZ acknowledge a grant No: 41/E-336/SPUB/SP/2019 from the Polish Ministry of Science and Higher Education.

\section{Authors' contributions}

J.M. performed all experiments, assisted in MRI, analyzed of blood samples, wrote manuscript

T.Z. performed magnetic resonance measurements, analyzed of MRI scans, wrote methods section concerning MRI

A.K. analyzed of MRI scans, wrote methods section concerning MRI

K.Z. assisted in conducting the experiment and analysis of blood samples

S.J. supervised of MRI scanning

A.C. provided input and advice during the experiment; especially provided advice on sex checked and unification of offspring

J. S. Provided input and assistance as needed; especially provided advice and input on the experiment in terms of tissue collection and on the resulting manuscript; wrote manuscript

\section{Ethics approval and conflict of interests}

The animal experiments were approved by the Animal Ethics Committee at the Poznan University of Life Sciences

\section{Conflict of interests}

There is no conflict of interests.

\section{Tables}

Table 1. Products and their nutritional values of CAF diet composition 
Nutritional values per $100 \mathrm{~g}$ of product

\begin{tabular}{lllllll}
\hline Products & Company & $\begin{array}{l}\text { Energy } \\
(\mathrm{kcal})\end{array}$ & $\begin{array}{l}\text { Fat } \\
(\mathrm{g})\end{array}$ & $\begin{array}{l}\text { Cabrohydrates } \\
(\mathrm{g})\end{array}$ & $\begin{array}{l}\text { Protein } \\
(\mathrm{g})\end{array}$ & Salt $(\mathrm{g})$ \\
\hline Cheese & Mlekpol & 347.0 & 27.0 & 0.0 & 26.0 & 1.2 \\
\hline Dried sausages & Tarczyński & 538 & 47.0 & 4.8 & 24.0 & 3.1 \\
\hline Biscuits & Krakuski & 440.0 & 13.0 & 71.0 & 8.9 & 1.1 \\
Chocolate & E. Wedel & 501.0 & 28.0 & 52.0 & 6.0 & 0.1 \\
\hline Peanut butter & Nusskati & 563.0 & 37.0 & 49.0 & 6.5 & 0.1 \\
\hline Chips & Lays & 536.0 & 33.0 & 52.0 & 5.9 & 1.1 \\
Cookies & E. Wedel & 358.0 & 7.0 & 70.0 & 3.2 & 0.2 \\
\hline Pate & Duda & 259.0 & 23.0 & 3.2 & 9.8 & 1.9 \\
Jam & Lowicz & 142.0 & 0.5 & 35.0 & 0.5 & 0.0 \\
Choccolate & & & & & & \\
bars & Nesstle & 488.0 & 21.6 & 68.5 & 4.1 & 0.5 \\
Dried bananas & Bakalland & 530.0 & 34.0 & 50.0 & 6.0 & 0.0 \\
\hline Muffins & & 431.0 & 24.0 & 47.0 & & 0.6
\end{tabular}

\section{Figures}

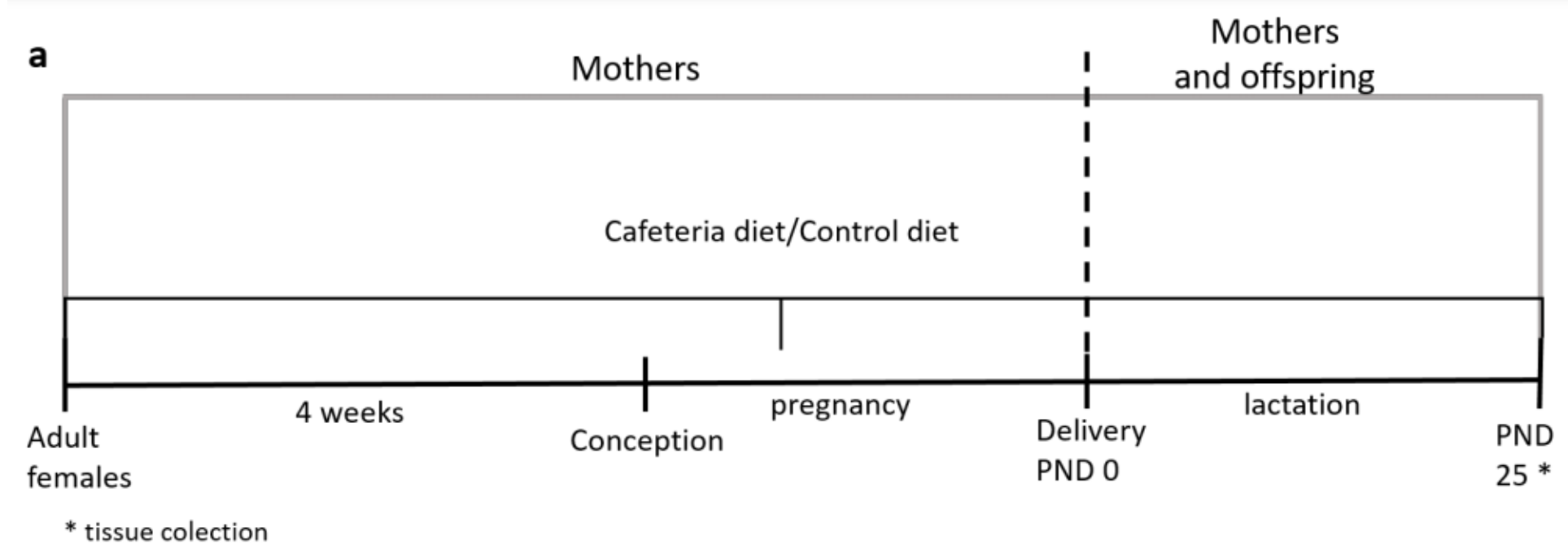

Figure 1

Experimental design After 4 weeks of feeding with cafeteria (CAF) or control (C) diets adult female rats were fertilized $(n=3)$. Administration of CAF diet was contained during pregnancy and lactation until postnatal day 25 (PND 25). 

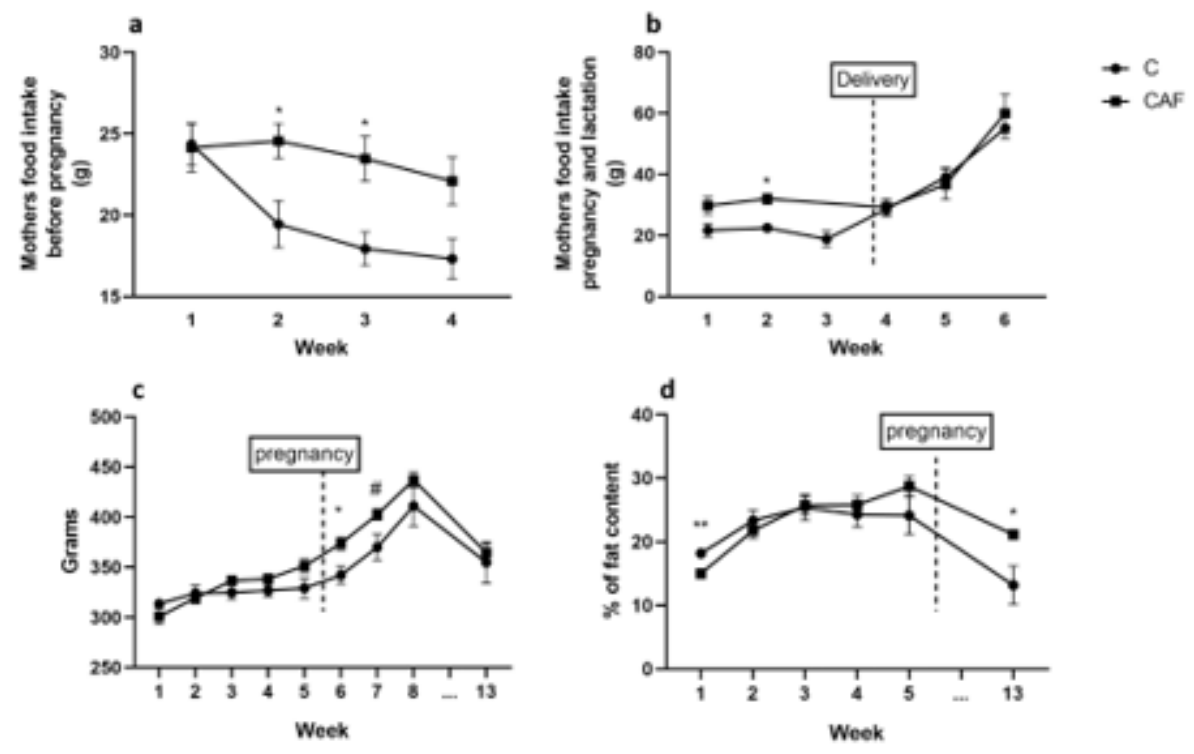

Figure 2

Mothers food intake before pregnancy and during pregnancy and lactation and maternal body weight and fat content (a) Weekly average of food intake in CAF and C groups; at 2 and 3 weeks CAF mothers had a significantly higher food intake ( $p$ 's< 0.05); (b) Weekly average of food intake during pregnancy and lactation. At 2nd week CAF mothers had significantly higher food intake ( $p<0.05)$; (c) Mothers weight in weekly time points: before pregnancy, during pregnancy and at the end of experiment; (d) Percentage of fat content in mothers measured weekly before pregnancy and at the end of experiment; black square CAF females, black dot $-C$ females $\left({ }^{*} p<0.05 ;{ }^{*} p<0.01 ; \# p=0.06\right) ; n=3$ mothers/group.
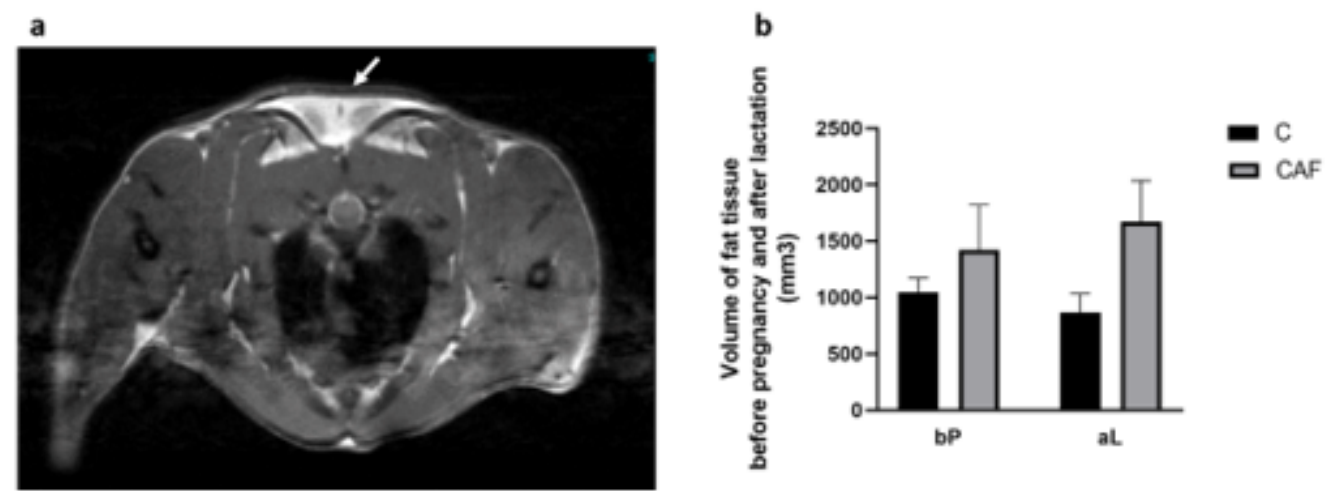

\section{Figure 3}

Magnetic resonance image (a) A representative magnetic resonance image from $\mathrm{C}$ group; white arrow point area of measurement of fat tissue; (b) volume of fat tissue measured before pregnancy and after lactation; $b P$ - before pregnancy $(p=0.42), a L-$ after lactation $(p=0.12)$ 

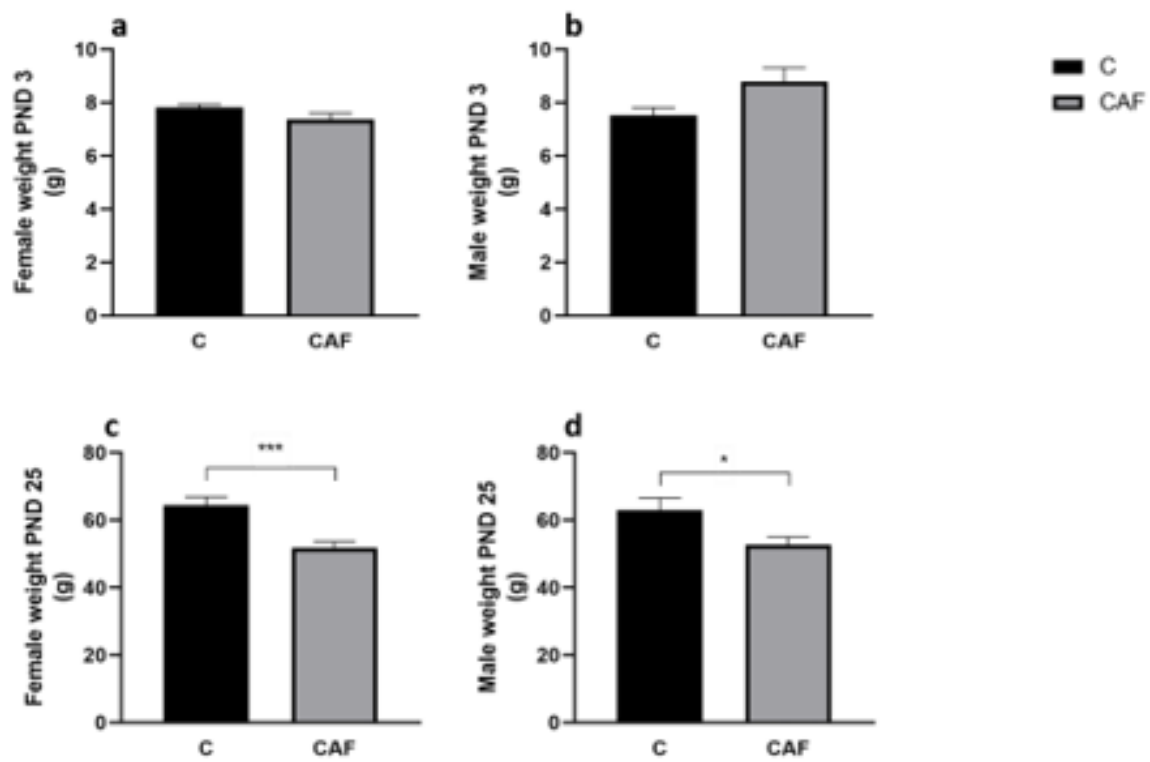

Figure 4

Weight of offspring at PND 3 and PND 25 (a) Comparison of weight of female offspring at PND 3 between CAF and C groups ( $p$ 's=0.11); (b) Comparison of weight of male offspring on PND 3 ( $p$ 's=0.11); (c) Comparison of weight of female offspring on PND 25 (***p's<0.001; females $n=9-13 /$ group); (d) Comparison of weight of male offspring on PND 25 ( ${ }^{*} p ' s<0.05$; males $n=7-10 /$ group); $C$ - control group, $\mathrm{CAF}$ - cafeteria group
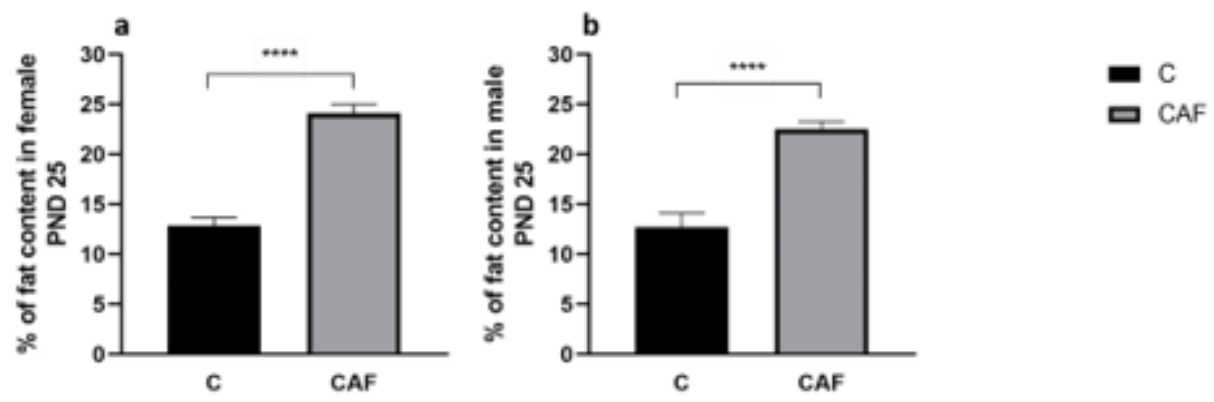

Figure 5

Percentage of fat content in offspring on PND 25 (a) Percentage of fat content in female offspring on

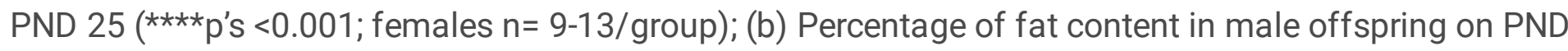
25 (****p's $<0.001$; males $n=7-10 /$ group); $C$ - control group, CAF - cafeteria group 

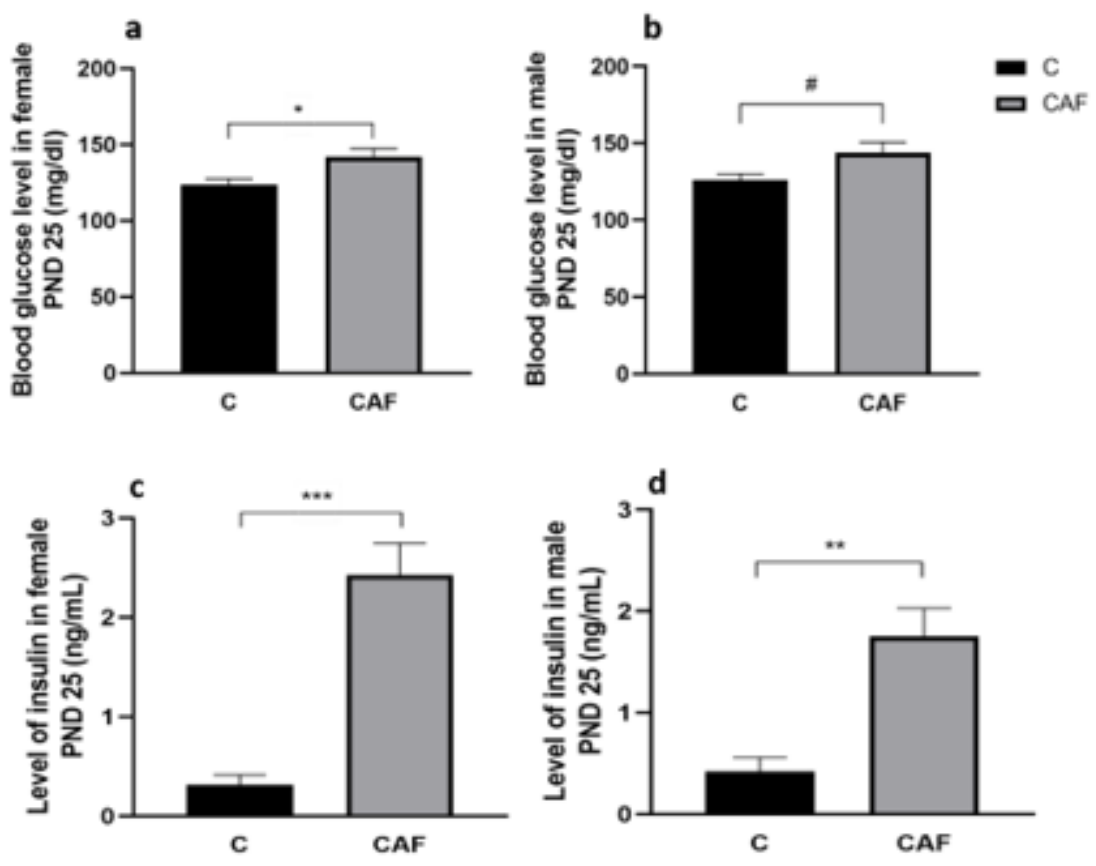

Figure 6

Metabolic status in offspring on PND 25 (a) Blood glucose level in female offspring ( ${ }^{*} p$ 's<0.05); (b) Blood glucose level in male offspring ( $\# p=0.07)$; (c) Blood serum level of insulin in female offspring $(p<0.005)$; (d) Blood serum level of insulin in male offspring ( $\left.{ }^{* \star} p<0.01\right)$; $C$ - control group, CAF - cafeteria group; $n=$ $5 /$ sex/group
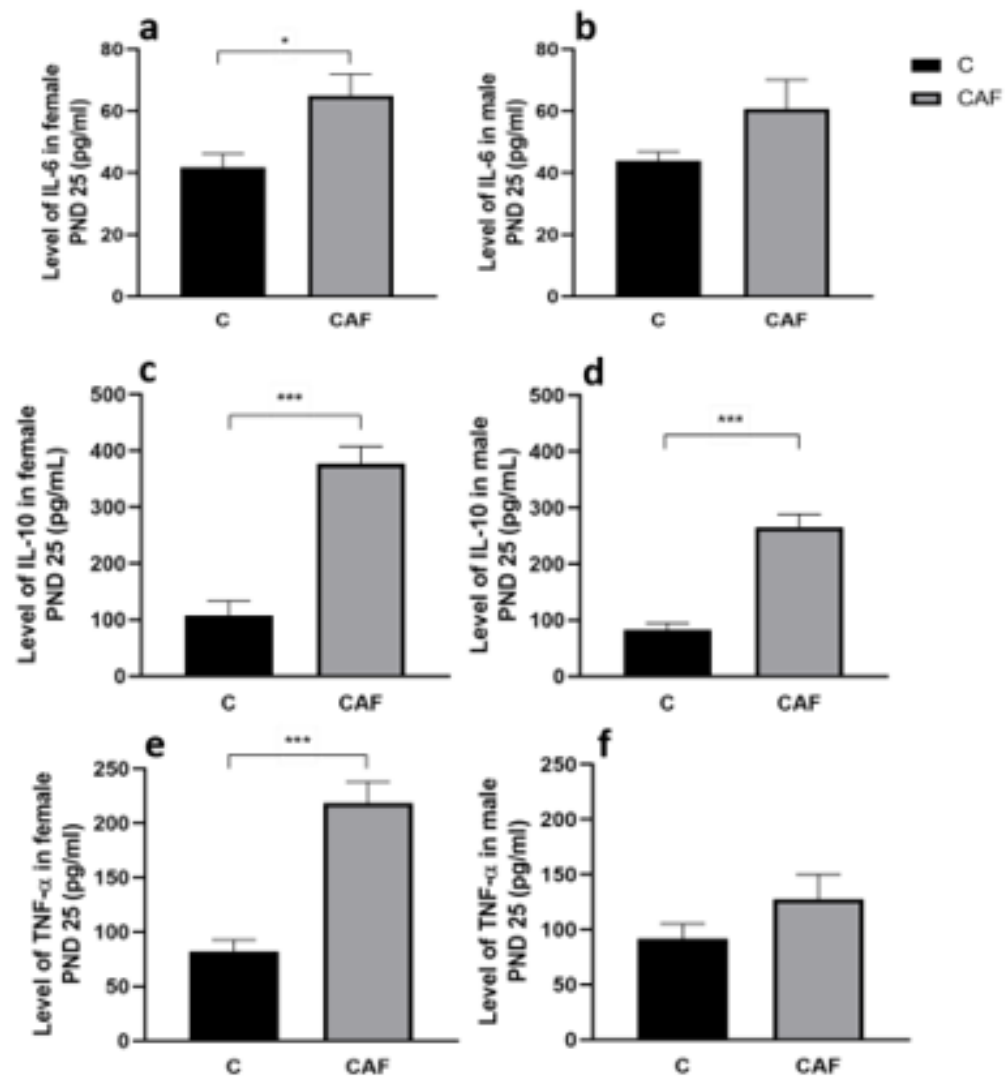


\section{Figure 7}

Inflammation status in offspring on PND 25 (a) Level of interleukin 6 (IL-6) in female offspring ( $\left.{ }^{*}{ }^{\prime} s<0.05\right)$; (b) IL-6 level in male offspring ( $\left.p=0.12\right)$; (c) Level of interleukin 10 (IL-10) in female offspring $\left({ }^{\star \star \star} p<0.005\right)$; (d) Blood serum level of IL-10 in male offspring ( $\left.p<0.005\right)$; (e) Level of tumor necrosis factor alpha (TNF-a) in female offspring ( $\left.{ }^{* \star *} p<0,005\right)$; (f) Level of TNF-a in male offspring $(p=0.20)$. C control group, $\mathrm{CAF}$ - cafeteria group; $n=5 /$ sex/group for all measurements 